\title{
ON THE INTERSECTIONS OF THE COMPONENTS OF A DIFFERENCE POLYNOMIAL
}

\author{
RICHARD M. COHN
}

The purpose of this note is to prove the following theorem:

Solutions common to two distinct components ${ }^{1}$ of the manifold of a difference polynomial annul the separants of the polynomial.

We begin by considering a field $K$, not necessarily a difference field, and a set of polynomials $F_{1}, F_{2}, \cdots, F_{p}$ in $K\left[u_{1}, \cdots, u_{q}\right.$; $\left.x_{1}, \cdots, x_{p}\right]$, the $u_{i}$ and $x_{j}$ being indeterminates, where for each $j, j=1, \cdots, p-1, F_{j}$ is free of the $x_{k}, k>j$. We shall show that any zero of $F_{1}, \cdots, F_{p}$ which annuls no formal partial derivative $\partial F_{j} / \partial x_{j}$ belongs to just one component of $\left\{F_{1}, \cdots, F_{p}\right\}_{0 .}{ }^{2}$ Furthermore, this component is of dimension $q$.

Proof. Let $u_{i}=\gamma_{i}, i=1, \cdots, q ; x_{j}=\alpha_{j}, j=1, \cdots, p$, be a zero of $F_{1}, \cdots, F_{p}$ which annuls no $\partial F_{j} / \partial x_{j}$. If $\gamma_{1}^{\prime}, \cdots, \gamma_{q}^{\prime} ; \alpha_{1}^{\prime}, \cdots, \alpha_{p}^{\prime}$ is a zero of $F_{1}, \cdots, F_{p}$ which specializes to $\gamma_{1}, \cdots, \gamma_{q} ; \alpha_{1}, \cdots, \alpha_{p}$, then this zero too annuls no $\partial F_{j} / \partial x_{j}$. It follows from this that $\alpha_{1}^{\prime}$ is algebraic over $K\left(\gamma_{1}^{\prime}, \cdots, \gamma_{q}^{\prime}\right)$, and that for each $k, 1<k \leqq p, \alpha_{k}^{\prime}$ is algebraic over $K\left(\gamma_{1}^{\prime}, \cdots, \gamma_{q}^{\prime} ; \alpha_{1}^{\prime}, \cdots, \alpha_{k-1}^{\prime}\right)$. This implies that a component of the manifold of $\left\{F_{1}, \cdots, F_{p}\right\}_{0}$ containing $\gamma_{1}, \cdots, \gamma_{q}$; $\alpha_{1}, \cdots, \alpha_{p}$ is of dimension at most $q$.

We let $u_{i}=t_{i}+\gamma_{i}, i=1, \cdots, q ; x_{j}=\alpha_{j}+h_{j}, j=1, \cdots, p$. Here the $t_{i}$ denote new indeterminates and the $h_{j}$ certain formal series in positive integral powers of the $t_{i}$. We shall show that the $h_{j}$ may be so chosen that these substitutions annul $F_{1}, \cdots, F_{p}$. In fact, the lemma proved in [3] shows that for each $k, 1 \leqq k \leqq p$, we may annul $F_{k}$ by substitutions $u_{i}=t_{i}+\gamma_{i}, i=1, \cdots, p, x_{j}=s_{j}+\alpha_{j}, j<k, x_{k}=\alpha_{k}$ $+h_{k}^{\prime}$, where the $s_{j}, j=1, \cdots, p$, are new indeterminates, and $h_{k}^{\prime}$ is a formal series in positive integral powers of the $t_{i}$ and $s_{j}, j<k$. For $h_{1}$ we take $h_{1}^{\prime}$; for $h_{2}$ we take the result of replacing $s_{1}$ in $h_{2}^{\prime}$ by $h_{1}^{\prime}$, and so on.

With the $h_{j}$ as described let $\Sigma$ denote the set of polynomials in $K\left[u_{1}, \cdots, u_{q} ; x_{1}, \cdots, x_{p}\right]$ which are annulled by the above substitutions. Evidently $\Sigma$ is a prime p. i. (polynomial ideal). Its dimen-

Received by the editors April 17, 1954.

1 The term "component," not previously defined for difference manifolds, is to have the expected meaning: a component is a maximal irreducible submanifold of a manifold. For definitions of other terms and symbols see $[2 ; 3 ; 4]$.

${ }^{2}$ As in Chapter IV of [1] this notation indicates the perfect polynomial ideal generated by $F_{1}, F_{2}, \cdots, F_{p}$. 
sion is $q$ and the $u_{i}$ form a parametric set. For evidently $\Sigma$ can contain no polynomial in the $u_{i}$ alone, while the conclusion of the preceding paragraph but one shows that its dimension cannot exceed $q$. The result of that paragraph also shows that no component of $\left\{F_{1}, \cdots, F_{p}\right\}_{0}$ can properly contain the manifold of $\Sigma$, for then its dimension would exceed $q$. Hence this manifold is itself a component of $\left\{F_{1}, \cdots, F_{p}\right\}_{0}$.

Let $\mathcal{X}$ be a component of $\left\{F_{1}, \cdots, F_{p}\right\}_{0}$ which contains $\gamma_{1}, \cdots, \gamma_{q} ; \alpha_{1}, \cdots, \alpha_{p}$, and let $\Lambda$ be the prime p. i. in $K\left[u_{1}, \cdots, u_{q}\right.$; $\left.x_{1}, \cdots, x_{p}\right]$ whose manifold is $\mathcal{X}$. We must show that $\Lambda$ is $\Sigma$. If $\Lambda$ is of dimension 0 then, because $\Sigma$ vanishes for a zero of $\Lambda$, and every zero must be a generic zero, $\Sigma$ is contained in $\Lambda$. Since the manifolds of both are components of the same manifold, it follows that $\Lambda=\Sigma$ (and that $q=0$ ). We suppose that $\Lambda$ is of positive dimension, and that $\Lambda$ and $\Sigma$ are distinct. Then, since $\Lambda$ cannot contain $\Sigma$, there is a polynomial $P$ in $\Sigma$ which is not in $\Lambda$. Then $\Lambda$ possesses a zero not annulling $P$ of the form

$$
\begin{array}{rlrl}
u_{i} & =\gamma_{i}+g_{i}, & i & =1, \cdots, q ; \\
x_{i} & =\alpha_{j}+f_{j}, & j & j, \cdots, p,
\end{array}
$$

where the $g_{i}$ and the $f_{j}$ are series in positive integral powers of a parameter $t$.

It is evident that (1) is a zero of $F_{1}, \cdots, F_{p}$. We may also obtain a zero of these polynomials of the form

$$
\begin{aligned}
u_{i} & =\gamma_{i}+g_{i}, & i & =1, \cdots, q ; \\
x_{j} & =\alpha_{j}+f_{i}^{\prime}, & j & =1, \cdots, p,
\end{aligned}
$$

where the $f_{j}^{\prime}$ are again series in positive integral powers of $t$, and each $f_{j}^{\prime}$ is obtained by replacing the $t_{i}, i=1, \cdots, p$, in $h_{j}$ by the corresponding $g_{i}$. It is evident from the manner of formation of (2) that it is a zero of $\Sigma$.

We replace the $u_{i}$ in $F_{1}$ by $\gamma_{i}+g_{i}, i=1, \cdots, q$. There results a polynomial $\bar{F}_{1}$ in $x_{1}$ with coefficients power series in $t . \bar{F}_{1}$ vanishes, but its formal derivative $d \bar{F}_{1} / d x_{1}$ does not, when we put $t=0, x_{1}=\alpha_{1}$. It follows that there is a unique series $f_{1}^{\prime \prime}$ in positive integral powers of $t$ such that $x_{1}=\alpha_{1}+f_{1}^{\prime \prime}$ is a solution of $\bar{F}_{1}=0$. We now replace the $u_{i}, i=1, \cdots, q$, and $x_{1}$ in $F_{2}$ by $\gamma_{i}+g_{i}$ and $\alpha_{1}+f_{1}^{\prime \prime}$ respectively to obtain a polynomial $\bar{F}_{2}$ in $x_{2}$ with coefficients power series in $t$. As before, we see that $\bar{F}_{2}=0$ possesses a solution $x_{2}=\alpha_{2}+f_{2}^{\prime \prime}$, where $f_{2}^{\prime \prime}$ is a series in positive integral powers of $t$. This series is unique. Continuing in this way we find uniquely determined $f_{j}^{\prime \prime}, j=1, \cdots, p$, which are series in positive integral powers of $t$ such that $u_{i}=\gamma_{i}+g_{i}$, 
$i=1, \cdots, q ; x_{j}=\alpha_{j}+f_{j}^{\prime \prime}, j=1, \cdots, p$, is a zero of $F_{1}, \cdots, F_{p}$.

The uniqueness of the $f_{j}^{\prime \prime}$ shows that (1) and (2) are identical. Hence (1) annuls $\Sigma$, and, in particular, it annuls $P$. We have thus obtained a contradiction. This completes the proof of our statement concerning the zeros of $F_{1}, \cdots, F_{p}$.

Now let $\mathcal{F}$ be a difference field and $A$ a polynomial of $\mathcal{F}\left\{y_{1}, \cdots, y_{n}\right\}$. We shall prove the theorem stated at the beginning of this note. We may suppose that a transform of some $y_{i}$, say of $y_{n}$, appears effectively in $A$. Let $y_{i}=\alpha_{i}, i=1, \cdots, n$, be a zero of $A$. It will suffice to assume that the $\alpha_{i}$ are not a zero of the $y_{n}$-separant of $A$ and show that this implies that only one component of the manifold of $A$ contains the $\alpha_{i}$.

It is evident that the $\alpha_{i}$ must annul just one irreducible factor, say $F$, of $A$, and do not annul the $y_{n}$-separant of $F$. Hence we need merely show that the $\alpha_{i}$ are contained in only one component of the manifold of $F$. We shall suppose that this is not so and obtain a contradiction. We assume first that $F$ is of equal order and effective order in $y_{n}$.

Let $\mathscr{X}_{1}$ and $\mathscr{X}_{2}$ denote two distinct components of the manifold of $F$, each containing the $\alpha_{i}$. Let $\Sigma_{1}$ and $\Sigma_{2}$ denote the corresponding reflexive prime difference ideals. We denote by $h$ the order of $F$ in $y_{n}$. Since the $\alpha_{i}$ do not annul the $y_{n}$-separant of $F, y_{1}, \cdots, y_{n-1}$ constitute a parametric set for both $\Sigma_{1}$ and $\Sigma_{2}$, and these ideals are both of order $h$ in $y_{n}$.

We choose an integer $m$ such that the first $m+1$ polynomials of a characteristic sequence of $\Sigma_{1}$ do not constitute the beginning of a characteristic sequence of $\Sigma_{2}$. Let $\Sigma_{1 m}$ and $\Sigma_{2 m}$ denote the sets consisting of those polynomials of $\Sigma_{1}$ and $\Sigma_{2}$ respectively which involve the $y_{n} \widehat{k}, 0 \leqq k \leqq m+h$, and a finite subset $S$ of the $y_{i j}, i<n . S$ is to include all those $y_{i j}, i<n$, which appear effectively, or whose transforms appear effectively, in $F, F_{1}, \cdots, F_{m}$ or in the first $m+1$ polynomials of a characteristic sequence of $\Sigma_{1}$ or in the first $m+1$ polynomials of a characteristic sequence of $\Sigma_{2}$.

$\Sigma_{1 m}$ and $\Sigma_{2 m}$ may be regarded as prime.i.'s in the Pring $\mathcal{f}\left[S, y_{n 0}, y_{n 1}, \cdots, y_{n, m+h}\right]$. The $y_{i j}$ of $S$ and the $y_{n k}, k<h$, constitute a parametric set for both $\Sigma_{1 m}$ and $\Sigma_{2 m}$. Let $s$ denote the number of indeterminates in this parametric set.

Our earlier result concerning polynomial ideals shows that there is a unique component $\mathcal{H}$ of the manifold of $\left\{F, F_{1}, \cdots, F_{m}\right\}_{0}$, regarded as an ideal of $\mathcal{F}\left[S, y_{n 0}, y_{n 1}, \cdots, y_{n, m+h}\right]$, which contains the zero $y_{i j}=\alpha_{i j}$ of this ideal. The dimension of $\mathcal{X}$ is $s$, for $s$ corresponds to $q$ of the earlier proof.

Now both $\Sigma_{1 m}$ and $\Sigma_{2 m}$ contain $\left\{F, F_{1}, \cdots, F_{m}\right\}_{0}$, while both have 
the zero $y_{i j}=\alpha_{i j}$. Hence their manifolds are in $\mathscr{H}$. Since their manifolds are of dimension $s$, however, they must coincide with $\mathcal{X}$. Hence $\Sigma_{1 m}$ and $\Sigma_{2 m}$ are identical. But $m$ was chosen so that $\Sigma_{1 m}$ contains a polynomial which is not in $\Sigma_{2 m}$, namely one of the first $m+1$ polynomials of a characteristic sequence of $\Sigma_{1}$. We have obtained a contradiction. This completes the proof of the theorem in the case that $F$ is of equal order and effective order in $y_{n}$.

If the order of $F$ in $y_{n}$ exceeds its effective order by $d>0$, we replace each $y_{n k}$ in $F$ by $z_{k-d}$, where $z$ is a new indeterminate, and subscripts attached to $z$ denote transforming. $F$ goes into an irreducible polynomial $\bar{F}$ which is of equal order and effective order in $z$.

Evidently each component $\overline{\mathscr{H}}$ of the manifold of $\bar{F}$ corresponds to a unique component $\mathcal{X}$ of the manifold of $F$, and, conversely, each component of the manifold of $F$ is obtained from a unique component of the manifold of $\bar{F}$. The correspondence may be described as follows: each solution in $\overline{\mathscr{X}}$ is obtained from a solution in $\overline{\mathscr{X}}$ by leaving unchanged the elements assigned as values to $y_{1}, \cdots, y_{n-1}$, and assigning to $y_{n}$ an element whose $d$ th transform is the element assigned as the value of $z$ in $\overline{\mathscr{X}}$. This correspondence carries solutions common to two components of the manifold of $F$ into solutions common to two components of the manifold of $\bar{F}$. Solutions annulling the $y_{n}$-separant of $F$ correspond to solutions annulling the $z$-separant of $\bar{F}$.

The preceding proof shows that the theorem stated at the beginning of this note holds for $\bar{F}$. The correspondence just described shows that its truth for $\bar{F}$ implies its truth for $F$. Hence it is true in general.

\section{REFERENCES}

1. J.F. Ritt, Differential algebra, Amer. Math. Soc. Colloquium Publications, vol. 33, 1950.

2. R. M. Cohn, Manifolds of difference polynomials, Trans. Amer. Math. Soc. vol. 64 (1948) pp. 133-172.

3. - A note on the singular manifolds of a difference polynomial, Bull. Amer. Math. Soc. vol. 54 (1948) pp. 917-922. 530.

4. — Extensions of difference fields, Amer. J. Math. vol. 74 (1952) pp. 507-

RUTGERS UNIVERSITY 